\title{
Stable Human-Robot Interaction Control for Upper-limb Rehabilitation Robotics
}

\author{
Juanjuan Zhang, Chien Chern Cheah and Steven H. Collins
}

\begin{abstract}
Research on rehabilitation robotics has been rising as a substitute to human practice to help neuro-damaged patients to restore impaired or lost functionalities. Most control methods for rehabilitative robotics do not consider the closedloop system stability in presence of uncertainty of nonlinear dynamics, and conflicting movements between patient and robots. In this paper, we present a theoretical framework which allows rigorous stability analysis of human-robot interaction in rehabilitative robotic system. Position-dependant stiffness and desired trajectory are proposed to resolve the possible conflicts in motions between patient and robot. The proposed method also realizes the assist-as-needed policy and possesses the ability to be customized for operations during different stages of patient recovery. In addition, the proposed controller handles human-robot interactions in such a way that correct movements are encouraged and incorrect ones are suppressed to make the training process more effective. Experimental results are presented to illustrate the performance of the controller.
\end{abstract}

\section{INTRODUCTION}

Stroke and traumatic brain injury affect millions of people each year. Many of the survivors suffer from severe neurological damage causing losses of motion control, i.e., paralysis. In 1949, Donald Hebb raised the idea of synaptic plasticity [1], which states that neurons that "fire" together, "wire" together, and simultaneous activation of cells leads to increases in synaptic strength between those neurons and therefore learning and relearning of human movements. Therefore, repeated and concurrent happening of human intentions to move and limb movements is the key to recovery. Therapies realizing this process have been started around 1950s. However, clinical practice is inefficient due to its great labor-intensity [2] and bad repeatability. To cover these issues, research on therapeutic robotics or rehabilitative robotics has started since early 1990s [3].

Isolated robot control theories with systematic stability analysis have already been established in the literature. However, different from the traditional robotic systems, control of rehabilitative systems is not always a typical servo-mechanism [4]. Besides stability, there are two main objectives of a rehabilitative controller, encouraging human intentions to move and ensuring correct movements. Operation of rehabilitative robotics includes two major stages. At the robot-dominant stage, human signals are either missing or untrustworthy, rehabilitative robotics work just like the assistive ones by treating human actions as disturbances and traditional control methods are applicable. The robot-complementing stage, on the contrary, stands for a scenario when motion-related

J. Zhang and C. C. Cheah are with School of Electrical and Electronic Engineering, Nanyang Technological University, Emails: jzhang12, ecccheah@e.ntu.edu.sg. S. H. Collins, is with Department of Mechanical Engineering, Carnegie Mellon University, Email: stevecollins@cmu.edu neurological connection is restored but weak. Kazerooni et. al pointed out in [4] that different from the typical servomechanism, human intentions and movements should be encouraged and complemented rather than rejected for this stage. Traditional control methods are therefore not feasible. The primary control paradigm in robotic therapies, just like physician based practices, is the active assist exercise [5], in which the patient initiates a movement, or a predetermined desired trajectory is known. Then the device will help to move the limbs. Existing assistive controllers of rehabilitative systems are mostly directly or indirectly derived from motion and impedance control [6]. Some are model-based controllers with [7] or without force feedback [4]. Others are non-modelbased methods with less predictable interactions between patients and devices and thus less control of the training progress [8]. More early devices employ motion-only-based control and handle interactions by disturbance rejection or uncertain modelling, using either linear [2] or non-linear force field [9], [10].

However, in all these designs, stability of the systems are not systematically solved with non-linearity, uncertainty, changing human dynamics or interaction forces. Besides, conflicting movements between human and robot may result in large tracking errors which lead to a large control force or even instability.

In this paper, we propose an adaptive controller for the upper-limb rehabilitative robotic systems, which handles human-robot interaction in such a way that correct human movements are encouraged while incorrect and conflicting movements are compensated or disregarded to ensure the efficiency of the training process. A theoretical framework allowing rigorous stability analysis of human-robot interaction in rehabilitative robotic system is presented. Positiondependant stiffness and desired trajectory are proposed to resolve the possible conflicts in motions between patients and robots. The proposed method also realizes the assistas-needed policy, with customizability based on different patients and stages to cope with the patient cooperative training [11] at various recovery stages. Tests were conducted to illustrate the performance of the proposed method.

\section{Dynamics of UpPeR-Limb Therapeutic Robotics}

The dynamics of an upper-limb rehabilitative robotic system consisting of a n-DOF robot manipulator connected to a human arm at the end can be expressed as

$$
M(q) \ddot{q}+\left(\frac{1}{2} \dot{M}(q)+S(q, \dot{q})\right) \dot{q}+g(q)=\tau+J(q)^{T} f_{h} .
$$


where $M(q) \in \mathfrak{R}^{n \times n}$ is the manipulator inertia matrix that is symmetric and positive definite on, $q \in \mathfrak{R}^{n}$, the joint variable vector; $\left(\frac{1}{2} \dot{M}(q)+S(q, \dot{q})\right) \dot{q} \in \mathfrak{R}^{n}$ is the centripetal and Coriolis torques in which $S(q, \dot{q})$ is skew-symmetric such that $y^{T} S(q, \dot{q}) y=0$, for any $y \in \mathfrak{R}^{n}[12] ; g(q) \in \mathfrak{R}^{n}$ denotes the gravitational torques; $\tau \in \mathfrak{R}^{n}$ stands for the control input torques applied at the manipulator joints. $J(q)^{T} f_{h} \in \mathfrak{R}^{n}$ denotes the torques due to the interactions between system and user, and $J(q) \in \mathfrak{R}^{n \times n}$ is the Jacobian transferring task space forces to joint space torques.

\section{Human-Robot Interaction Involved Control}

We begin by identifying the various operation modes of the rehabilitative robotics.

Based on the required capabilities of the rehabilitative robotics, the controller to be proposed should realize three basic operation modes of the system, 1) a human-dominant mode in which the patient movements are trusted and encouraged thus only slightly interfered by the robot system; 2) a robot-dominant mode in which patient motion control is not reliable and desired movements are accomplished mostly with robotic assistance, and 3) a safety-stop mode in which the system is stopped to ensure human system.

In human-dominant mode, human motion control is partially restored and it is important that the affected human limb gains enough exercise, i.e., limb free motion should be allowed to a great extent. Meanwhile, certain level of speed regulation is beneficial to avoid abnormal movements. Both of these two actions can strengthen the accuracy and repeatability of human motion control. On the contrary, in robot-dominant mode, assistance from the robot system is thus always necessary to either finish the task or put the human limb back to the correct track, both by moving the system closer to a desired trajectory. Trajectory tracking with regulation on both position and speed is suitable for this stage. Besides these two normal operation modes, sometimes, the system may drift so much that the position errors are too big and resulted control torques may incur damages to human body. In this case, the robotic system should be ceased and the faster the system moves, the faster it should be stopped. Thus, damping control should be employed here.

In practice, it is reasonable for a normal rehabilitative robot to able to work in all the three modes, determined by its end-effector's instant position. As shown in Fig. 1, when the end-effector is close to the desired trajectory, it is in human-dominant region (H-DR); when it is in the robotdominant region ( $\mathrm{R}-\mathrm{DR}$ ), robotic help is provided to push it back into H-DR; however, when the end-effector is drifted in to the outer safety-stop region (S-SR), the whole system is stopped. The additional transitional subregion of S-SR (TS-SR) appears between R-DR and stable S-SR (SS-SR) to ensure the smoothness of the controller, whose emergence and necessity will be detailed later.

In order to fit in the conditions of various patients and recovery stages, the widths of H-DR and R-DR are expected to be adjustable. At the early stage of the therapies when human movements are unreliable, H-DR should be shrinked to a

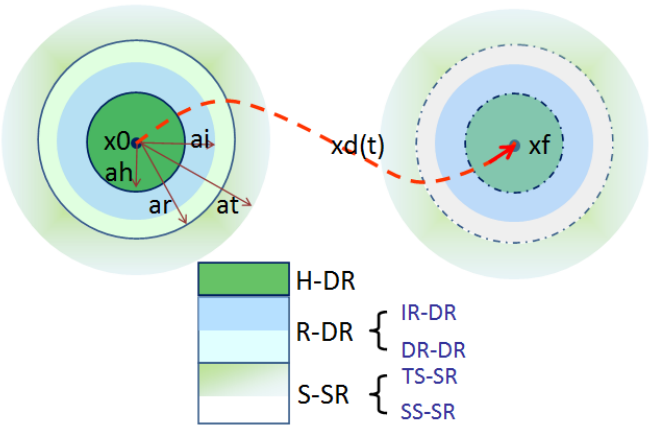

Fig. 1: Operation regions around the desired trajectory

very small area so that robot-assistance is constantly present. The rehabilitative robot actually works as an assistive one in this case. Otherwise, when human motion control has been mostly recovered, H-DR should be enlarged to allow more freedoms in human movements.

\section{A. Proposed Controller}

Besides realization of the three operation regions mentioned in the previous section, the desired controller should be capable to deal with system uncertainties. Thus, a controller is proposed as

$$
\begin{gathered}
\tau=-\mathcal{M}^{T}(x) J^{T}(q) k_{p}(\delta x) \delta x-K_{s} s \\
+Y_{d}\left(q, \dot{q}, \dot{q}_{r}, \ddot{q}_{r}\right) \hat{\theta}+J^{T}(q) C_{x}\left(f_{h}, \Delta x, s\right),
\end{gathered}
$$

where position error $\Delta x=x(t)-x_{\text {des }}(t)$, with robot endeffector position $x(t)=\left[x_{1}(t), x_{2}(t), \ldots, x_{m}(t)\right]^{T}$ and desired position $x_{\text {des }}(t)=\left[x_{d e s, 1}(t), x_{d e s, 2}(t), \ldots, x_{d e s, m}(t)\right]^{T} ; \delta x$ represents the modified position error, i.e.,

$$
\begin{aligned}
\delta x & =x(t)-x_{d}(t)=x(t)-w(\Delta x) x_{d e s}(t) \\
& =x(t)-w\left(x(t)-x_{\text {des }}(t)\right) x_{\text {des }}(t),
\end{aligned}
$$

where $w(\Delta x)$ stands for a weight vector to be detailed later and $x_{d}(t)$ is the weighted desired trajectory; $k_{p}(\delta x)$ denotes the proportional control gain, i.e., the stiffness; $K_{s}$ denotes the sliding gain. Controller $J^{T}(q) C_{x}\left(f_{h}, \Delta x, s\right)$ is used to handle human-robot interaction and will be detailed later. A modifier matrix

$$
\mathcal{M}(x)=I-J^{+}(q) A(x) J(q)
$$

is added to eliminate the use of $\ddot{x}$ in controller, $J^{+}(q)$ denotes the pseudo-inverse of $J(q)$ and matrix $A(x)$ shall be detailed later. The regressor $Y_{d}\left(q, \dot{q}, \dot{q}_{r}, \ddot{q}_{r}\right) \in \mathfrak{R}^{n \times p}$ is defined by [12]:

$$
M(q) \ddot{q}_{r}+\left[\frac{1}{2} \dot{M}(q)+S(q, \dot{q})\right] \dot{q}_{r}+g(q)=Y_{d}\left(q, \dot{q}, \dot{q}_{r}, \ddot{q}_{r}\right) \theta
$$

where $\theta$ denotes a vector of unknown parameters, and the estimated parameters $\hat{\theta}$ is updated by:

$$
\dot{\hat{\theta}}=-L Y_{d}^{T}\left(q, \dot{q}, \dot{q}_{r}, \ddot{q}_{r}\right) s
$$

with a positive-definite and non-singular square matrix gain $L$. The sliding vector $s$ is proposed as

$$
\begin{gathered}
s=\dot{q}-\dot{q}_{r} \\
=\mathcal{M}^{-1}(x)\left[\dot{q}-J^{+}(q) \dot{x}_{d}(t)+\alpha J^{+}(q) k_{p}(\delta x) \delta x\right]
\end{gathered}
$$

where $\alpha$ is a constant gain.

At a time instant $t$ when the robot-human cooperative system is in action, the work space is divided into three portions based on the distance of $x(t)$ and $x_{d e s}(t)$ : H-DR with radius $a_{h}, \mathrm{R}-\mathrm{DR}$ with outer radius $a_{r}$ and S-SR. These three regions 
move along $x_{\text {des }}(t)$ together as shown in Fig. 1 .

To meet the operation requirements, firstly, the proportional control term $-J^{T}(q) k_{p}(\delta x) \delta x, k_{p}(\delta x)$ should be strictly asserted to zero in H-DR, non-zero in the R-DR. Besides, with the increase of $\delta x$ inside R-DR, it is reasonable for $k_{p}(\delta x)$ to ramp up to rapidly restore the system back to H-DR. However, the value of $-J^{T}(q) k_{p}(\delta x) \delta x$ will grow to a point when the control torque becomes damaging to human body. Similar issues happens to term $-K_{s} s$. Concern for human safety therefore nurtures the necessity of S-SR, in which $k_{p}(\delta x)$ is again set to zero to cease stiffness control and damping will be introduced to stop the motion as detailed later. Two functions are used to meet these conditions:

- The position-dependant weight vector $w(\Delta x)$ is added to the desired trajectory $x_{d e s}(t)$ such that the weighted function as in (3) meets the requirements

$$
w(\Delta x) x_{\text {des }}(t)=\left\{\begin{array}{cc}
x_{\text {des }}(t), & x \in \text { H-DR\& R-DR }, \\
{\left[0, x_{\text {des }}(t)\right],} & x \in \text { TS-SR } \\
0, & x \in \text { SS-SR } .
\end{array}\right.
$$

Definition of $w(\Delta x)$ is based on two region functions:

$$
\begin{aligned}
& f_{w 1}(\Delta x)=\frac{\left(x_{1}-x_{\text {des }, 1}\right)^{2}}{a_{t}^{2}}+\ldots+\frac{\left(x_{m}-x_{\text {des }, m}\right)^{2}}{a_{t}^{2}}-1=\frac{\|\Delta x\|^{2}}{a_{t}^{2}}-1 \leq 0, \\
& f_{w 2}(\Delta x)=\frac{\left(x_{1}-x_{d e s, 1}\right)^{2}}{a_{r}^{2}}+\ldots+\frac{\left(x_{m}-x_{d e s, m}\right)^{n}}{a_{r}^{2}}-1=\frac{\|\Delta x\|^{2}}{a_{r}^{2}}-1 \leq 0,
\end{aligned}
$$

where $a_{r}$ is the outer radius of R-DR to be determined later; $a_{t}=a_{r}+h_{t}$ stands for outer radius of TS-SR, and $h_{t}$ is the width of the TS-SR to be designed. The continuous and differentiable weight factor is then chosen as $w(\Delta x)$

$$
=\left\{\begin{array}{cc}
0, & f_{w 1}(\Delta x) \geq 0, \\
1-\frac{\left\{\left[f_{w 1}(\Delta x)\right]^{N}-\left(\frac{a_{r}^{n}}{a_{t}^{n}}-1\right)^{N}\right\}^{N}}{\left(\frac{a_{r}^{n}}{a_{t}^{n}}-1\right)^{N^{2}}}, & f_{w 2}(\Delta x)>0, f_{w 1}(\Delta x)<0, \\
1, & f_{w 2}(\Delta x) \leq 0,
\end{array}\right.
$$

which smoothly changes from 0 to 1 when system transits from outside to inside S-SR. Let $A(x)$ be a matrix such that:

$$
A(x)=\left[\begin{array}{ccc}
\frac{\partial w}{\partial x_{1}} x_{\text {des }, 1} & \cdots & \frac{\partial w}{\partial x_{m}} x_{d e s, 1} \\
\vdots & \ddots & \vdots \\
\frac{\partial w}{\partial x_{1}} x_{d e s, m} & \cdots & \frac{\partial w}{\partial x_{m}} x_{d e s, m}
\end{array}\right] .
$$

Define a vector $s_{q}=\dot{q}-J^{+}(q) \dot{x}_{d}+\alpha J^{+}(q) k_{p}(\delta x) \delta x$. From Eq. (3), $s_{q}$ can be rewritten as

$$
s_{q}=\left[I-J^{+}(q) A(x) J(q)\right] \dot{q}-J^{+}(q) \dot{x}_{f}+\alpha J^{+}(q) k_{p}(\delta x) \delta x
$$

with $\dot{x}_{f}=w(\Delta x) \dot{x}_{d e s}+A(x) \dot{x}_{d e s}$. Now, the sliding vector can be expressed in terms of $s_{q}$ as $s=\mathcal{M}(x)^{-1} s_{q}$. Then, by Eq. (4) and (6), $s$ can be written as

$$
s=\dot{q}-\mathcal{M}(x)^{-1} \times\left[J^{+}(q) \dot{x}_{f}-\alpha J^{+}(q) k_{p}(\delta x) \delta x\right] .
$$

Now, with this weighted desired trajectory above, considering the fact that $A(x)=0$ and thus $\mathcal{M}(x)=I$, for $w=0 \& 1$, i.e., in H-D, R-DR and SS-SR, the damping control part is

$$
\begin{aligned}
& -K_{s} s=-K_{s} \mathcal{M}^{-1}(x)\left[\dot{q}-J^{+}(q) \dot{x}_{d}+\alpha J^{+}(q) k_{p}(\delta x) \delta x\right] \\
& =\left\{\begin{array}{cc}
-K_{s}\left[\dot{q}-J^{+}(q) \dot{x}_{d e s}\right], & \text { H-DR, } \\
-K_{s}\left[\dot{q}-J^{+}(q) \dot{x}_{d e s}+\alpha J^{+}(q) k_{p}(\delta x) \delta x\right], & \text { R-DR, } \\
-K_{s} \mathcal{M}^{-1}(x)\left[\dot{q}-J^{+}(q) \dot{x}_{d}\right], & \text { TS-SR, } \\
-K_{s} \dot{q}, & \text { SS-SR. }
\end{array}\right.
\end{aligned}
$$

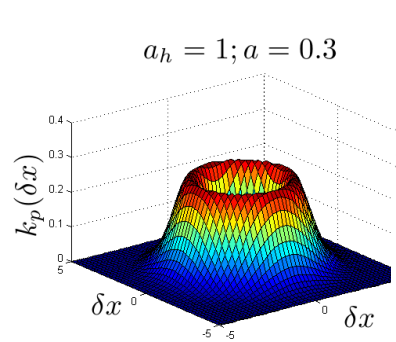

(a) $k_{p}$ vs $\delta x$ 3D view

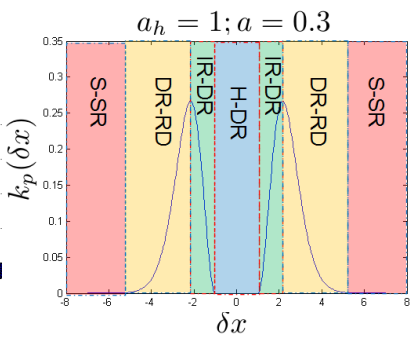

(b) $k_{p}$ vs $\delta x$ intersection
Fig. 2: Designed variable stiffness $k_{p}(\delta x)$

which implements speed control in H-DR and TS-SR, trajectory tracking in R-DR and damping in SS-SR.

- The position-dependant stiffness $k_{p}(\delta x)$ such that

$$
k_{p}(\delta x)=\left\{\begin{array}{cc}
0, & x \in \mathrm{H}-\mathrm{DR}, \\
\text { positive values, } & x \in \mathrm{R}-\mathrm{DR} \\
0, & x \in \mathrm{S}-\mathrm{SR}
\end{array}\right.
$$

To obtain a continuous and differentiable stiffness that meets these requirements, an auxiliary function is first defined as

$$
f_{p}(\delta x)=1-\exp \left[-a\left(\|\delta x\|^{2}-a_{h}^{2}\right)\right],
$$

and the position dependant stiffness is accordingly

$$
k_{p}(\delta x)=k_{1}\left[\max \left(0, f_{p}(\delta x)\right)\right]^{2} \exp \left[-a\left(\|\delta x\|^{2}-a_{h}^{2}\right)\right],
$$

in which $a$ and $k_{1}$ are positive constants of design, and $a_{h}$ is the radius of H-DR as mentioned earlier. An illustration of this function is available in Fig. 2 with $a_{h}=1, a=0.3$. It is observed that there are two subregions for R-DR, an increasing R-DR (IR-DR) and a decreasing R-DR (DR-DR). The outer radius of the former, $a_{i}$, is adjustable by the

$$
a_{i}=\left\{\delta x: \frac{\partial k_{p}(\delta x)}{\partial \delta x}=0 \& \delta x>a_{h}\right\}=f\left(a, a_{h}\right) .
$$

The outer radius of DR-DR and whole R-DR, $a_{r}$, is also controllable by $k_{1}, a$ and $a_{h}$. Assuming a controller minimum distinguishable signal $p$, i.e., $x \equiv 0$, if $x<p$, then, $a_{r}$ is determined by $a_{r}=\left\{\delta x: k_{p}(\delta x)=p \& \delta x>a_{i}\right\}$.

Note that H-DR provides the patients with opportunity to exercise and R-DR guarantees human action correctness and reinforces human motion control. Thus, the existence of of both regions is a key feature to the rehabilitative process and the relative size of them should reflects the recovery status of patients. When human movement is less reliable, $a_{h}$ should be set smaller to ensure less human free motion and more robotic assistance; when human gains better motion control, $a_{h}$ should be set larger to allow more exercise and little robot help as needed to further boom the recovery. When $a_{h}$ is set to zero, the system is in full robot-dominant stage, which works for robot-assisting stage of the therapy.

With the $k_{p}(\delta x)$ defined above, stiffness control part is

$$
-J^{T}(q) k_{p}(\delta x) \delta x=\left\{\begin{array}{cc}
0, & \text { if } x \in \mathrm{H}-\mathrm{DR}, \\
-J^{T}(q) k_{p}(\delta x) \delta x, & \text { if } x \in \mathrm{R}-\mathrm{DR} \\
0, & \text { if } x \in \mathrm{S}-\mathrm{SR}
\end{array}\right.
$$

which realizes proportional control in R-DR only.

With stiffness and desired trajectory defined above, the control law is proposed as Eq. (2). Note that the inclusion of $J^{+}(q)$ and $\mathcal{M}(x)=\left[I-J^{+}(q) A(x) J(q)\right]$ in the controller 


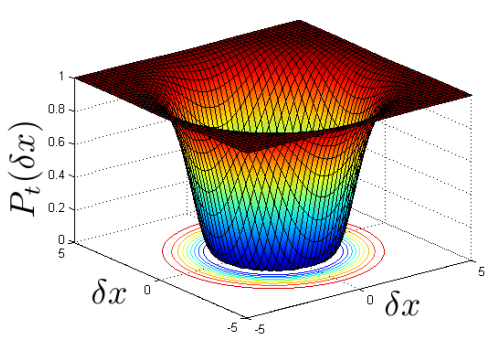

Fig. 3: Pseudo-energy shape with $a_{r}=2$

potentially causes issues at the singularity points of $J^{+}(q)$. This can be handled by asserting $k_{p}(\delta x)=0$ in the S-SR when $J^{+}(q)$ is singular. It is realized by selecting a $x_{d e s}(t)$ with all singular points $\in \mathrm{S}$-SR, i.e.,

$$
\tau=-K_{s} \dot{q}+Y_{d}\left(q, \dot{q}, \dot{q}_{r}, \ddot{q}_{r}\right) \hat{\boldsymbol{\theta}}+J^{T}\left[C x\left(\Delta x, f_{h}, s\right)+f_{h}\right],
$$

damping control is employed, and the system will be stopped. Substituting Eq. (2) into Eq. (1), the closed-loop system dynamics becomes

$$
\begin{aligned}
& M(q) \dot{s}+\left[\frac{1}{2} \dot{M}(q)+S(q, \dot{q})\right] s+\mathcal{M}(x)^{T} J^{T}(q) k_{p}(\delta x) \delta x \\
& +K_{s} s+Y_{d}\left(q, \dot{q}, \dot{q}_{r}, \ddot{q}_{r}\right) \Delta \theta=J^{T}(q)\left[C_{x}\left(f_{h}, \Delta x, s\right)+f_{h}\right],
\end{aligned}
$$

where $\Delta \theta=\theta-\hat{\theta}$. Let the output be $y=s$. Without interaction-related controller, i.e., $C_{x}\left(f_{h}, \Delta x, s\right)=0$, the time integral of the inner product between $y$ and Eq. (10) yields

$$
\int_{0}^{t} y(\varsigma)^{T} J^{T}(\varsigma) f_{h}(\varsigma) d \varsigma=
$$

$$
\int_{0}^{t}\left\{s(\varsigma)^{T} M(q(\varsigma)) \dot{s}(\varsigma)+s(\varsigma)^{T}\left[\frac{1}{2} \dot{M}(\varsigma)+S(\varsigma)\right] s(\varsigma)\right.
$$

$\left.+\left[\mathcal{M}(\varsigma)^{-1}\right]^{T}\left[\dot{q}(\varsigma)-J^{+}(\varsigma) \dot{x}_{d}(\varsigma)\right)\right]^{T} \mathcal{M}(\varsigma)^{T} J^{T}(\varsigma) k_{p}(\varsigma) \delta x(\varsigma)$

$+\left[\mathcal{M}(\varsigma)^{-1} \alpha J^{+}(\varsigma) k_{p}(\varsigma) \delta x(\varsigma)\right]^{T} \mathcal{M}(\varsigma)^{T} J^{T}(\varsigma) k_{p}(\varsigma) \delta x(\varsigma)$

$$
\left.+s(\varsigma)^{T} K_{s} s(\varsigma)+s(\varsigma)^{T} Y_{d}(\varsigma) \Delta \theta(\varsigma)\right\} d \varsigma
$$$$
=\underbrace{V(t)-V(0)}_{\text {Stored Energy }}+\underbrace{\int_{0}^{t} W(\varsigma) d \varsigma}_{\text {Dissipated Energy }},
$$

where $V=\frac{1}{2} s^{T} M(q) s+P_{t}(\delta x)+\frac{1}{2} \Delta \theta L^{-1} \Delta \theta$, with a pseudo-potential function $P_{t}(\delta x)$ expressed as $P_{t}(\delta x)=$ $\frac{1}{6 a} k_{1}\left[\max \left(0, f_{p}(\delta x)\right)\right]^{3}$ and

$$
W=s^{T} K_{s} s+\alpha \delta x^{T} k_{p}^{2}(\delta x) \delta x .
$$

Then, since instant stored energy $V(t)$ in the system is composed of quadratic forms $\frac{1}{2} \Delta \theta L^{-1} \Delta \theta, s^{T} M(q) s$ and $P_{t}(\delta x)$, which is illustrated in Fig. 3, it is clear that

$$
V(t) \geq 0 \text {. }
$$

Next, both of the two items of the RHS of Eq. (11) are positive, i.e., the energy dissipation rate $W(s, \dot{q}) \geq 0$ is positive. Hence, input $J^{T}(q) f_{h}$ and output $y=s$ have passive relationship. With $f_{h}=0$, energy stored will not increase. However, due to the existence of human-robot interaction, the term $s^{T} J^{T}(q) f_{h} \neq 0$. Thus, a interaction controller $J^{T}(q) C_{x}\left(f_{h}, \Delta x, s\right)$ needs to be introduced such that $s^{T} J^{T}(q)\left[C_{x}\left(f_{h}, \Delta x, s\right)+f_{h}\right] \leq 0$ to ensure the negativeness of

$$
-W^{\prime}=\dot{V}=-W+s^{T} J^{T}(q)\left[C_{x}\left(f_{h}, \Delta x, s\right)+f_{h}\right] .
$$

\section{B. Interaction-related Controller}

To achieve the non-positiveness of $s^{T} J^{T}(q)\left[C_{x}\left(f_{h}, \Delta x, s\right)+\right.$ $\left.f_{h}\right]$, the term is firstly written as

$s^{T} J^{T}(q)\left[C_{x}\left(f_{h}, \Delta x, s\right)+f_{h}\right]=s_{x}^{T}\left[C_{x}\left(f_{h}, \Delta x, s\right)+f_{h}\right]$, in which

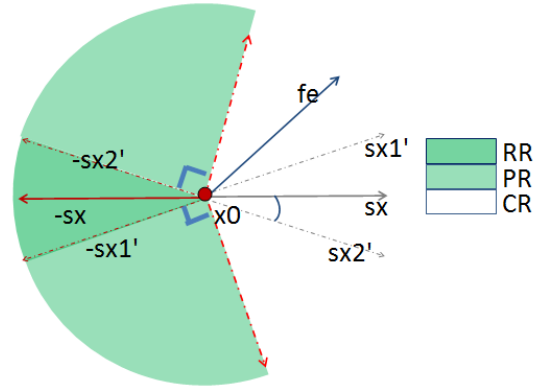

Fig. 4: Interaction force handling regions illustration $s_{x}=J(q) s=J(q) \mathcal{M}^{-1}(x) s_{q}$ $=\left\{\begin{array}{cc}\dot{\delta} x+\alpha k_{p}(\delta x) \delta x, & \mathrm{H}-\mathrm{DR}, \mathrm{R}-\mathrm{DR} \& \mathrm{SS}-\mathrm{SR}, \\ J(q) \mathcal{M}^{-1}(x) s_{q}, & \text { TS-SR. }\end{array}\right.$

Research work [13] has shown that human central nervous system may use a composite variable composed of tracking errors $\delta x$ and their temporal derivatives $\delta \dot{x}$ in motion control as either a error prediction or a criterion to be minimized. By a similar means, in a robotic rehabilitative system, the controlled interaction received by the robot should be fully responsible for minimizing this variable. For the controller proposed, the variable is represented by the task space sliding vector $s_{x}$, a combination of the transformed position and velocity errors.

Next, in S-SR where the system should stop, $f_{h}$ is directly canceled. In H-DR, human movements are fairly reliable, and the controller is varied in three sectors based on the directional difference between $-s_{x}$ and $f_{h}, \gamma$, as demonstrated in Fig. 4 to encourage human movements. When $\|\gamma\|$ is smaller than a threshold $\beta$, which is adjustable in $\left[0,90^{\circ}\right.$ ), human motion is trusted, thus the interaction is retained [retaining region $(R R)$ ]. If $f_{h}$ is out of this area but still maintains an angle difference of less than $90^{\circ}$ with one RR edge, $-s_{x 1}^{\prime}$ or $-s_{x 2}^{\prime}$, it is projected to whichever edge that is closer [projecting region $(P R)$ ]. Beyond these two regions, $f_{h}$ is considered wrongly orientated and is canceled [cancelling region $(C R)$ ]. Finally, R-DR serves as a transition area.

According to the discussions above, the interaction-related controller should be developed such that

$$
\begin{gathered}
C_{x}\left(f_{h}, \Delta x, s\right)+f_{h}=\left\{\begin{array}{cl}
0, & x \in \mathrm{S}-\mathrm{SR}, \\
\mu_{s}(s) \mu_{x}(\Delta x) c_{x}\left(f_{h}\right), & x \in \mathrm{R}-\mathrm{DR}, \\
\mu_{s}(s) c_{x}\left(f_{h}\right), & x \in \mathrm{H}-\mathrm{DR},
\end{array}\right. \\
\text { where } c_{x}\left(f_{h}\right)=\left\{\begin{array}{cc}
f_{h}, & f_{h} \in \mathrm{RR}, \\
-s_{x}^{\prime}\left\|f_{h}\right\| \cos ^{2}(\|\gamma\|-\beta), & f_{h} \in \mathrm{PR}, \\
0, & f_{h} \in \mathrm{CR},
\end{array}\right.
\end{gathered}
$$

with $s_{x}^{\prime}$ equals to $s_{x 1}^{\prime}$ or $s_{x 2}^{\prime}$, whichever has a closer direction with $f_{h}$. The term

$$
\mu_{x}(\Delta x)=\sin ^{2}\left[\frac{\left(a_{r}-\|\Delta x\|\right) \pi}{2\left(a_{r}-a_{h}\right)}\right], \text { when } \Delta x \in \text { R-DR only, }
$$

is a position-error-based coefficient to ensure smooth transition between H-DR and S-SR; the saturation function

$$
\mu_{s}(s)=\left\{\begin{array}{cl}
1, & \left\|s_{x}\right\| \geq w_{s}, \\
\sin ^{2}\left(\frac{\left\|s_{x} \mid\right\| \pi}{2 w_{s}}\right), & \left\|s_{x}\right\|<w_{s}
\end{array}\right.
$$

is added as a second coefficient $c_{x}\left(f_{h}\right)$ to assert forcedominant controller when $s$ has norm bigger than $w_{s}$, a small 
positive constant of design, and sliding-vector-dominant controller otherwise, so that the controller is smoothed at $s=$ 0 . Note that instead of direct projection to $-s_{x}^{\prime}$ direction, a second order projection $\cos ^{2}(\bullet)$ is used to ensure its differentiability. Thus, the control result $C_{x}\left(f_{h}, \Delta x, s\right)+f_{h}$ is uniformly continuous on $s, \Delta x$ and also $f_{h}$, and the term

$$
s^{T}\left[C_{x}\left(f_{h}, \Delta x, s\right)+f_{h}\right]=\left\{\begin{array}{cc}
0, & x \in \mathrm{S}-\mathrm{SR}, \\
\mu_{s}(s) \mu_{x}(\Delta x) s_{x}^{T} c_{x}\left(f_{h}\right), & x \in \mathrm{R}-\mathrm{DR}, \\
\mu_{s}(s) s_{x}^{T} c_{x}\left(f_{h}\right), & x \in \mathrm{H}-\mathrm{DR},
\end{array}\right.
$$

where

$$
s_{x}^{T} c_{x}\left(f_{h}\right)=\left\{\begin{array}{cc}
-\left\|s_{x}\right\|\left\|f_{s}\right\| \cos (\gamma), & f_{h} \in \mathrm{RR}, \\
-\left\|s _ { x } \left|\left\|\mid f_{s}\right\| \cos (\beta) \cos ^{2}(\|\gamma\|-\beta),\right.\right. & f_{h} \in \mathrm{PR}, \\
0, & f_{h} \in \mathrm{CR} .
\end{array}\right.
$$

Given that $\beta<\frac{\pi}{2}$, the above equation is never greater than zero. Thus, Eq. (13) is proved to be negative semi-definite.

\section{Proof of Stability}

The convergence of the state variables depends on the robot end-effector position where it starts in or enters. Therefore, the stability of the system is investigated by operation regions. For all cases, the function $V$ represents a Lyapunovlike function for the closed-loop system with $V \geq 0$ and $\dot{V}=-W^{\prime} \leq 0$. Hence, it is clear that $V(t) \leq V(0)$, i.e., it is upper bounded. Since $V$ is positive-definite in $s$ and $\Delta \theta$, $s$ and $\Delta \theta$ are bounded.

\section{- Human-domiant \& Robot-dominant Regions}

If robot enters or starts in these regions, it is known that

$$
\begin{gathered}
s=\left[\dot{q}-J^{+}(q) \dot{x}_{d e s}+\alpha J^{+}(q) k_{p}(\delta x) \delta x\right], \\
w(\Delta x)=1, A(x)=0, \mathcal{M}^{-1}(x)=I, \\
V=\frac{1}{2} s^{T} M(q) s+P_{t}(\delta x)+\Delta \theta^{T} L^{-1} \Delta \theta,
\end{gathered}
$$$$
\dot{V}=-s^{T} K_{s} s-\alpha \Delta x^{T} k_{p}(\delta x)^{2} \delta x^{2}+s^{T} J^{T}(q)\left[C_{x}\left(f_{h}, \Delta x, s\right)+f_{h}\right]
$$

and the closed-loop dynamics is

$$
\begin{aligned}
& M(q) \dot{s}+\left[\frac{1}{2} \dot{M}(q)+S(q, \dot{q})\right] s+K_{s} s+J^{T}(q) k_{p}(\delta x) \delta x \\
& +Y_{d}\left(q, \dot{q}, \dot{q}_{r}, \ddot{q}_{r}\right) \Delta \theta-J^{T}(q)\left[C_{x}\left(f_{h}, \Delta x, s\right)+f_{h}\right]=0 .
\end{aligned}
$$

First, the boundedness of $s$ and $\Delta \theta$ are already established. Next, $k_{p}(\delta x) \delta x$ is bounded by definition. $\dot{q}_{r}=J^{+}(q)\left[\dot{x}_{f}-\right.$ $\left.\alpha k_{p}(\delta x) \delta x\right]$ is bounded if $\dot{x}_{f}=w(\Delta x) \dot{x}_{d e s}+A(x) \dot{x}_{d e s}$ is bounded, which is true if $\dot{x}_{d e s}$ is bounded. Then, according to Eq. (7), $\dot{q}$ is also bounded. Since $\dot{q}$ is bounded and $J(q)$ is composed of trigonometric functions of $q$, $\dot{x}=J(q) \dot{q}$ is bounded. By using $\mathcal{M}(x), \ddot{q}_{r}$ has been released from depending on $\ddot{x}$. Therefore, the boundedness of $\dot{x}, w(\Delta x), \dot{w}(\Delta x), \ddot{x}_{d e s}, \mathcal{M}(x)$ and $k_{p}(\Delta x) \Delta x$ suggests the boundedness of $\ddot{q}_{r}$. Therefore, regressor $Y_{d}\left(q, \dot{q}, \dot{q}_{r}, \ddot{q}_{r}\right)$ is bounded. Next,

$$
\begin{gathered}
\ddot{V}=-2 s^{T} K_{s} \dot{s}-2 \alpha k_{p}^{2}(\delta x) \delta x^{T} \dot{\delta} x \\
-2 \alpha \delta x^{T} k_{p}(\delta x) \delta x \frac{\partial k_{p}(\delta x)}{\partial \delta x} \dot{\delta} x+\dot{s}^{T} J^{T}(q)\left[C_{x}\left(f_{h}, \Delta x, s\right)+f_{h}\right] \\
+s^{T} \dot{J}^{T}(q)\left[C_{x}\left(f_{h}, \Delta x, s\right)+f_{h}\right]+s^{T} J^{T}(q)\left[\dot{C}_{x}\left(f_{h}, \Delta x, s\right)+\dot{f}_{h}\right]
\end{gathered}
$$

is bounded due to the boundedness of $\dot{s}, \delta x, \dot{\delta} x, k_{p}(\delta x)$, $\frac{\partial k_{p}(\delta x)}{\partial \delta x}$ and $\left[\dot{C}_{x}\left(f_{h}, \Delta x, s\right)+\dot{f}_{h}\right]$. According to Barbalet Lemma, $\dot{V} \rightarrow 0$, as $t \rightarrow \infty$, which implies

$$
\begin{gathered}
s \rightarrow 0, \text { as } t \rightarrow \infty, \text { and } \\
k_{p}(\delta x) \delta x \rightarrow 0, \text { as } t \rightarrow \infty .
\end{gathered}
$$

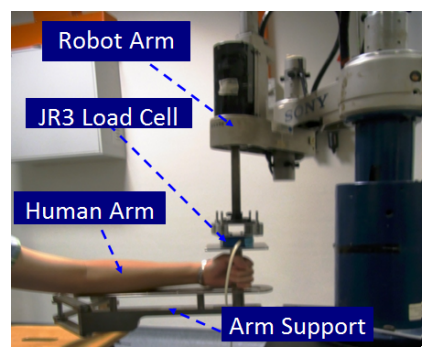

Fig. 5: 2-DOF planar upper-limb rehabilitative robotics Thus, it is clear that

$$
\dot{q}-J^{+}(q) \dot{x}_{d e s} \rightarrow 0, \text { as } t \rightarrow \infty \text {. }
$$

Combination of (17) and (18) yields,

$$
\left\{\begin{array}{c}
\dot{x} \rightarrow \dot{x}_{d e s}, \text { and }, \\
x \rightarrow \text { H-DR. }
\end{array}\right.
$$

Therefore, velocities of the end-effector inside H-DR or RDR converge to the desired ones $\dot{x}_{d e s}(t)$ and its position goes to H-DR with radius $a_{h}$ unless some strong external interferences force it into the S-SR, the analysis of which case is detailed in later.

\section{- Safety-stop Region}

If the robot end-effector enters this region accidentally or starts within it, it is known that

$$
k_{p}(\delta x) \delta x=0, C_{x}\left(f_{h}, \Delta x, s\right)+f_{h}=0 \text {, and } P_{t}(\delta x)=P_{t, s a t},
$$

with $P_{t, s a t}$ is the saturated $P_{t}(\delta x)$, which yields

$$
\begin{gathered}
s=\left\{\begin{array}{cc}
\mathcal{M}^{-1}(x)\left[\dot{q}-J^{+}(q) w(\Delta x) x_{d e s}\right], & \text { TS-SR, } \\
\dot{q}, & \text { SS-SR, }
\end{array}\right. \text { and } \\
\dot{V}= \\
\left\{\begin{array}{cl}
{\left[\mathcal{M}^{-1}\right]^{T}\left[\dot{q}-J^{+}(q) x_{d}\right]^{T} K_{s} \mathcal{M}^{-1}\left[\dot{q}-J^{+}(q) x_{d}\right],} & \text { TS-SR, } \\
-\dot{q}^{T} K_{s} \dot{q}, & \text { SS-SR. }
\end{array}\right.
\end{gathered}
$$

It means that damping is realized in SS-SR and velocity tracking towards $w(\Delta x) x_{\text {des }}$ in TS-SR. Therefore, when system is in SS-SR, the velocities converge to 0 rapidly and the system stops in this area unless it enters TS-SR before stopping. If by any means, system enters TS-SR, the endeffector velocity converges to the modified desired velocity while it approaches either R-DR or SS-SR.

In summary of the stability analysis, the robot end-effector always try to move in H-DR or stop in S-SR.

\section{Pilot Tests on Hardware}

The proposed controller was tested on a 2-DOF planer upperlimb rehabilitative robotics as shown in Fig. 5; The system is consisted of a 2-DOF planar robot manipulator connected to a passive arm supporter at the end. In between the robot end-effector and support, a JR3 6-axis load cell is installed to measure interaction forces. Three set of tests were conducted to test the performance of the controller. In all cases, these values are used: $k_{1}=1.5 e-2, a=3 e-2, K_{s}=3 e-6, L=$ $1 e-3 I, p=1 e-3, \beta=\frac{\pi}{6}$ and $h_{t}=0.01 \mathrm{~m}$.

In the first test, H-DR radius is set as $a_{h}=0 m$ to ensure full robotic dominance to test controller's assistive performance by investigating its trajectory following ability. $a_{i}=0.1914 m$ and $a_{r}=0.4799 m$ according to calculation. The actual trajectory of the system goes around the desired one as in Fig. 7(a) 

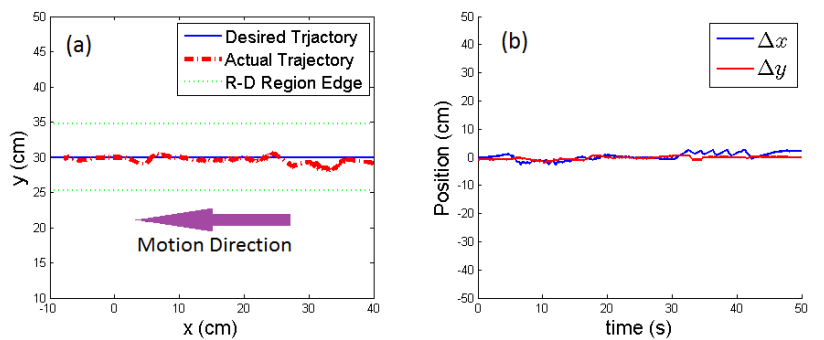

Fig. 6: Robot Assistive Tracking Results. (a) Assistive Trajectories (b) Position Errors vs. Time
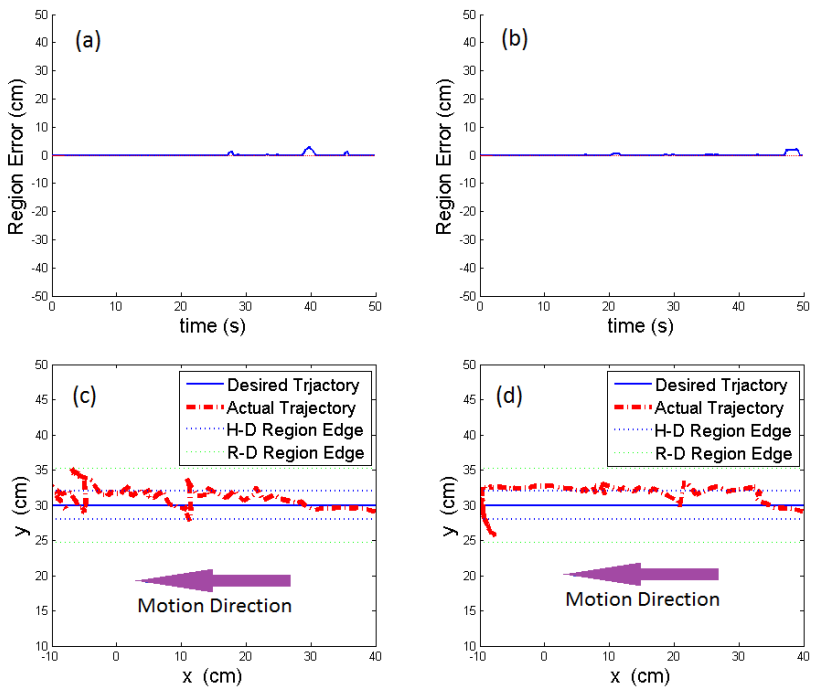

Fig. 7: Robot Rehabilitative Tracking Results. (a) Region Errors: $f_{h} \|$ Path (b) Region Errors: $f_{h} \perp$ Path (c) Trajectories: $f_{h} \|$ Path (d)Trajectories: $f_{h} \perp$ Path
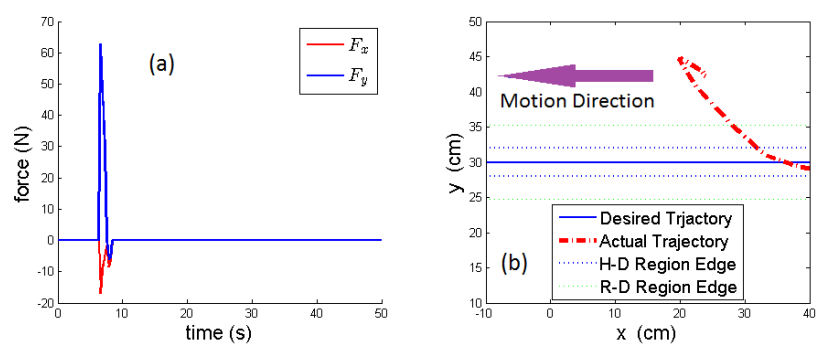

Fig. 8: Safety-stop performance. (a) Applied Force Spike (b) Trajectories

with position errors $\leq 3 \mathrm{~cm}$ as in Fig. 7(b). High oscillations of position errors are due to high frictions and unfiltered data. The second test aimed to investigate the region following and human action encouragement performance in H-DR when robot is working as a rehabilitative devices by setting $a_{h}=0.2 \mathrm{~m}$ with $f_{h}$ present, in which case, $a_{i}=0.2768 \mathrm{~m}$ and $a_{r}=0.5199 m$ accordingly. The regional errors, i.e., the distance from the system end-effector to H-DR, as shown in Fig. 8, say that the system stays within H-DR most of the time and possess the ability to be pulled back when it is not. Within H-DR, human user is given freedom to move. When human forces are perpendicular to the desired trajectory at the center of H-DR, system is pushed to and stays mostly at the edge of H-DR as seen in Fig. 8(d). When human forces are along the desired direction, end-effector is moving around H-DR center as demonstrated in Fig. 8(c).
Finally, the safety-stop capability of the controller is tested by starting the system in H-DR with $a_{h}=0.2 m$ and pushing the end-effector using a large human force spike as in Fig. 9(a) into the S-SR. It can be shown in Fig. 9(b) that the system stops in the S-SR after forced into this area as desired.

\section{CONCLUSIONS}

In this paper, the stability of upper-limb rehabilitative robotic system has been solved with system non-linearities, uncertainties and varying human-robot interactions. With the employment of position-dependant stiffness and positiondependant desired trajectory, the proposed controller possesses the capability of automatic smooth switch between different operation modes to realized "assist-as-needed" prodigy and customized region sizes to cope with various patients and their stages. With this controller, the rehabilitative system tries to move within H-DR or stop in S-SR to avoid damages to human. Pilot tests on hardware have been conducted to test the performance of the proposed controller, which provide a theoretical framework for upper-limb rehabilitative robotic system and can be extended to the analysis of other humanrobot interactive devices.

\section{REFERENCES}

[1] D. O. Hebb, The organization of behavior, Willy \& Sons, 1949.

[2] H. I. Krebs, N. Hogan, M. L. Aisen, and B. T. Volpe, "Robot-aided neurorehabilitation," IEEE trans. on rehabilitation engineering, vol.6, No.1, Mar. 1998

[3] N. Hogan, H. I. Krebs, J. Charnnarong, P. Srikrishna, and A. Sharon, "MIT-MANUS: A workstation for manual therapy and training," Proc. Int. Workshop Robot Human Communication, pp. 161-165, Sep. 1992.

[4] H. Kazerooni, J-L. Racine, L. Huang and R. Steger, "On the control of the Berkeley Lower Extremity Exoskeleton (BLEEX)," in Proc. IEEE Int. Conf. Robotics and Automation, Barcelona, Spain, April 2005.

[5] L. Marchal-Crespo, and D. J. Reinkensmeyer, "Review of control strategies for robotic movement training after neurologic injury," $J$. NeuroEngineering and Rehabilitation, Jun. 2009.

[6] J. R. Andres, and N. Hogan, "Impedance control as a framework for implementing obstacle avoidance in a manipulator," Control of Manufacturing Processes and Robotic Systems, ASME, New York, pp. 243-251, 1983.

[7] J. A. Saglia, N. G. Tsagarakis, J. S. Dai, and D. G. Caldwell, "Control strategies for ankle rehabilitation using a high performance ankle exerciser," Proc. IEEE Int. Conf. on Robotics and Automation,, pp 2221-2227, May 3-8, 2010.

[8] E. D. Fasse, and N. Hogan, "Control of physical contact and dynamic interaction," Proc. Int. Symp. on Robotics Research, Munich, 1995.

[9] L. Cai, A. Fong, C. Otoshi, Y. Liang, J. Burdick, R. Roy, and V. Edgerton, "Implications of assist-as-needed robotic step training after a complete spinal cord injury on intrinsic strategies of motor learning," J. Neuroscience, vol. 26, no. 41, pp. 10654-10658, 2006.

[10] M. Peshkin, D. A. Brown, J. J. Santos-Munné, A. Makhlin, E. Lewis, J. E. Colgate, J. Patton, and D. Schwandt, "KineAssist: A robotic overground gait and balance training device," Proc. IEEE 9th Int. Conf. on REhabilitation Robotics, pp. 241-246, 2005.

[11] R. Riener, L. Lunenburger, S. Jezernik, J. M. Anderschitz, G. Colombo, and V. Dietz, "Patient-cooperative strategies for robot-aided treadmill training: first experimental results," IEEE Trans. Neural Syst Rehabil Eng, vol. 13, no 3, pp. 380-394, 2005.

[12] S. Arimoto, Control Theory of Non-linear Mechanical Systems: A Passivity-based and Circuit-theoretic Approach, Clarendon Press, Oxford, 1996.

[13] S. Hanneton, A. Berthoz, J. Droulez, and J. J. E. Slotine, "Does the brain use sliding variables for the control of movements?" Biological Cybernetics, vol. 77, pp. 381-393, 1997. 